\title{
Prognostic significance of lymphocyte-to- monocyte ratio and CRP in patients with nonmetastatic clear cell renal cell carcinoma: a retrospective multicenter analysis
}

\author{
This article was published in the following Dove Press journal: \\ OncoTargets and Therapy \\ 9 May 2016 \\ Number of times this article has been viewed
}

\author{
Wen-Kai Xia \\ Xia Wu \\ Tang-Hong Yu \\ Yu Wu \\ Xia-Juan Yao \\ Hong $\mathrm{Hu}$ \\ Department of Nephrology, The \\ Affiliated Jiangyin Hospital of \\ Southeast University Medical \\ College, Jiangyin, Jiangsu, People's \\ Republic of China
}

Correspondence: Hong Hu Affiliated Jiangyin Hospital of Southeast University Medical College, 163 Shoushan Road, Jiangyin, Jiangsu 214400,

People's Republic of China Email huhong1523@I63.com
Department of Nephrology, The

Background: Inflammation has been reported to be involved in carcinogenesis and cancer progression. This study was designed to explore the prognostic significance of lymphocyte-tomonocyte ratio (LMR) and serum C-reactive protein (CRP) in nonmetastatic clear cell renal cell carcinoma (ccRCC) patients after treatment.

Methods: The retrospective study consisted of 985 patients with ccRCC who had undergone nephrectomy from 2005 to 2010 at multiple centers. The patients were divided into four groups using a quartile of LMR or CRP, and their associations with clinical characteristics and outcome were systematically estimated.

Results: Both low LMR and high CRP significantly diminished overall survival (OS) and metastasis-free survival (MFS) in patients with ccRCC. Further investigation indicated that LMR and CRP were independent prognostic factors of both OS and MFS. Integration of LMR and CRP into a predictive model, including significant variables in multivariate analysis, established a nomogram to predict accurately the 3- and 5-year survival for nonmetastatic patients with ccRCC.

Conclusion: LMR and CRP represent independent prognostic factors of OS and MFS for patients with ccRCC. Incorporation of LMR and CRP into the traditional TNM staging system may improve their predictive performance.

Keywords: C-reactive protein, lymphocyte-to-monocyte ratio, clear cell renal cell carcinoma, survival, nomogram

\section{Introduction}

Renal cell carcinoma (RCC) is a major malignancy of the kidneys worldwide. ${ }^{1}$ Clear cell renal cell carcinoma (ccRCC) is the most prevalent type of RCC and accounts for $>80 \%$ of all RCCs. ${ }^{2}$ Due to changes in the environment and lifestyle, the morbidity of RCC has presented an ascending trend over the past decades. ${ }^{3}$ Despite substantial advances in diagnosis, surgical resection, immunotherapy, and molecular-targeted therapy of RCC, the long-term survival is still unsatisfactory during follow-up owing to ineffective drug response, local relapse, and distant metastasis. ${ }^{4,5}$ At present, several prognostic models of clinical characteristics and different endpoints have been proposed to predict clinical outcomes for surgical patients with RCC, including the TNM staging system. ${ }^{6}$ However, precise prediction of individual disease prognosis remains difficult. Hence, there is an urgent need to find significant RCC markers together with traditional clinical characteristics to improve the prediction of prognosis. ${ }^{7}$

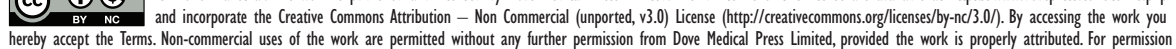
for commercial use of this work, please see paragraphs 4.2 and 5 of our Terms (https://www.dovepress.com/terms.php). 
Tumor-associated inflammation is commonly considered the seventh hallmark of cancer and is characterized by inflammatory cell infiltration and an activated stroma. ${ }^{8}$ Furthermore, patients with cancer frequently show the presence of systemic inflammatory responses, which are represented by surrogate peripheral blood-based parameters, such as neutrophil, monocytes, and C-reactive protein (CRP), showing favorable predictive probability of clinical outcomes of various solid cancers. ${ }^{9}$ Of these reported inflammatory factors, the neutrophil-tolymphocyte ratio has been shown to be a reliable indicator that can potentially predict the outcome in patients with RCC or those treated with chemotherapy for metastatic RCC. ${ }^{10-12}$

Tumor-associated macrophages, as well as surrogate peripheral blood cell counts, such as the lymphocyte-tomonocyte ratio (LMR), as potential markers for predicting survival of patients with cancer, including gastric cancer, ${ }^{13}$ colorectal cancer, ${ }^{14}$ and lung cancer, ${ }^{15}$ have been generally reported. The published data show a profound influence of decreased LMR on the survival of patients with RCC in a single center study. ${ }^{16-18}$

We conducted a multicenter study to further clarify the prognostic role of LMR on clinical outcomes of 958 patients with ccRCC. Furthermore, we developed a predictive model based on a combination of patients' age, tumor size, $\mathrm{T}$ and $\mathrm{N}$ stage, LMR, and CRP to predict the 3- or 5-year survival for nonmetastatic patients with ccRCC.

\section{Patients and methods}

\section{Patients}

All experimental procedures were approved by the Medical Ethics Committee of The Affiliated Jiangyin Hospital of Southeast University Medical College. Written consents were collected from patients at first follow-up.

This retrospective study enrolled 985 patients with newly diagnosed nonmetastatic ccRCC from 2005 to 2010 at the Jiangyin People's Hospital (Jiangsu, People's Republic of China), Hangzhou First People's Hospital (Zhejiang, People's Republic of China), Zhejiang Cancer Hospital (Zhejiang, People's Republic of China), and other hospitals in Jiangsu Province. Patients with non-ccRCC confirmed histopathologically, ccRCC patients with distant metastasis, a history of previous anticancer treatment, bilateral renal cancer, hematology disease, acute and chronic inflammation, hyperpyrexia, gastrobrosia, and abnormal routine laboratory tests at diagnosis were excluded. For each patient, the following clinical characteristics were obtained: sex, age at diagnosis, a history of smoking or drinking, tumor size, $\mathrm{T}$ and $\mathrm{N}$ stage, TNM stage, ${ }^{6}$ absolute lymphocyte and monocyte counts, and CRP. Histopathologic results of the tumor tissues were confirmed by experiential pathologists. Tumor size was marked as the longest diameter described in pathologic findings. The hematological and laboratory parameters were collected within 1-3 days of diagnosis.

\section{Blood sample analysis}

All peripheral blood was collected in ethylene diamine tetraacetic acid tubes within 1 week prior to treatment. Absolute lymphocyte and monocyte counts were measured by Sysmex XT-1800i Automated Hematology System (Sysmex, Shanghai, People's Republic of China). LMR were calculated from absolute blood cell counts. CRP (mg/L) was determined by an immunonephelometry.

\section{Follow-up}

Patients with nonmetastatic RCC were treated with partial or radical nephrectomy as appropriate in the hospitals. After surgery, each patient was routinely followed-up (every 6 months for the first 2 years and then annually) with physical examination, laboratory test, chest imaging, and abdominal ultrasound after surgery. Overall survival (OS) was defined as the time (in months) from diagnosis to death from all causes or censored at the last follow-up date. Metastasis-free survival (MFS) was defined as the time (in months) from diagnosis to the metastasis of radiologically or histologically confirmed distant metastases.

\section{Statistical analysis}

All statistical analysis was performed with SPSS 18.0 software (SPSS Inc., Chicago, IL, USA) and R software version 3.2.2 and the rms package (Institute for Statistics and Mathematics, Vienna, Austria). Comparison of categorical variables was handled by the Pearson $\chi^{2}$ test. The Kaplan-Meier method with log-rank test was applied to evaluate survival rates. We performed the Cox proportional hazards regression model in univariate analysis and thereafter the significant variables of univariate analysis were included into the multivariable analysis. Due to a lack of commonly approved cutoff value of LMR with skewed distribution, we used the quartile as the cutoff for discrimination. R software was used to establish a predictive model by "rms" package. The performance features of this model were confirmed by calibration plots. The predictive accuracy was determined by the Harrell's concordance index (c-index). $P<0.05$ was considered statistical significance.

\section{Results \\ Clinical characteristics}

A total of 985 patients with ccRCC were enrolled in this study. The detailed clinical characteristics of patients are summarized in Table 1. The median age at diagnosis was 55 years, which 


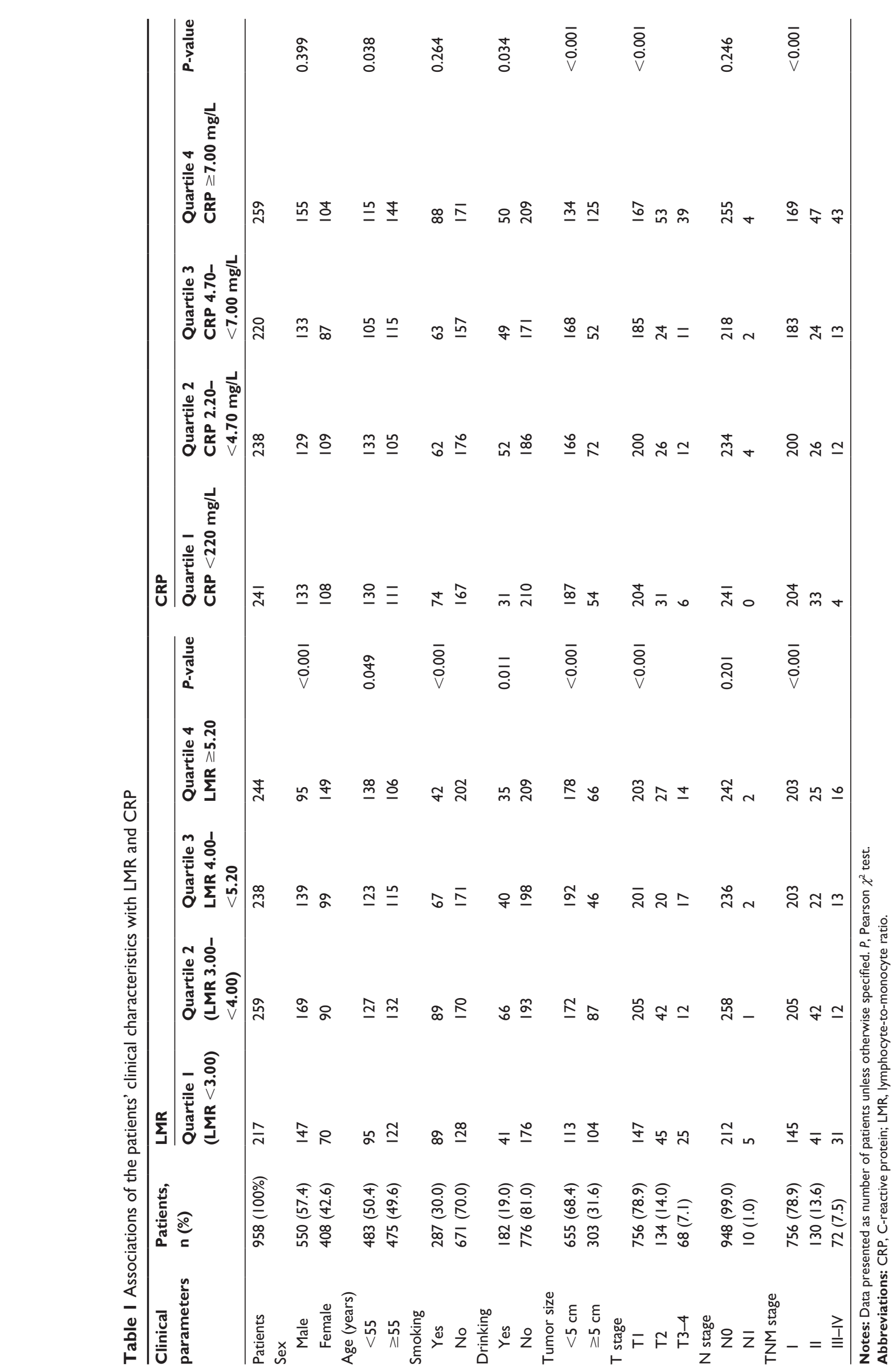


ranged from 21 to 81 years in the 550 males and 408 females. Median LMR and CRP were 4.00 (interquartile range: 3.00-5.20) and $4.70 \mathrm{mg} / \mathrm{L}$ (interquartile range: $2.20-7.00$ ), respectively. Median follow-up was 58 months, which ranged from 3 to 60 months. During follow-up period, 133 patients (13.9\%) died from all causes and 188 patients (19.6\%) experienced disease recurrence or distant metastasis.

As the distribution of LMR and CRP is not normal, we employed the quartile as endpoints to evaluate the associations of LMR and CRP with clinical characteristics. Our results (Table 1) showed that LMR was significantly associated with patients' sex, smoking, drinking, tumor size, T and TNM stage. The association of their diagnosed age and LMR was borderline significance $(P=0.049)$. Meanwhile, CRP at diagnosis was correlated with patients' age, drinking, tumor size, T and TNM stage but not related to sex, smoking, and $\mathrm{N}$ stage.

\section{Associations of LMR and CRP with patients' outcomes}

Survival curves and log-rank tests were used to investigate the associations of LMR and CRP with patients' survival. Both elevated CRP and decreased LMR were significantly associated with shorter OS and MFS (Figure 1, $P<0.001$ ). Furthermore, results from the univariate analysis revealed that LMR and CRP were prognostic factors of both OS and MFS as well as patients' age, tumor size, $\mathrm{T}$ and $\mathrm{N}$ stage (Table 2), but sex, and history of smoking and drinking had no prognostic significance for OS and MFS. According to our multivariate analysis, LMR, CRP, and tumor size were independent prognostic indicators for OS $(P<0.001$, hazard ratio $[\mathrm{HR}]=0.23$ for quartile $(\mathrm{Q}) 4 / \mathrm{Q} 1 ; P<0.001, \mathrm{HR}=3.44$ for

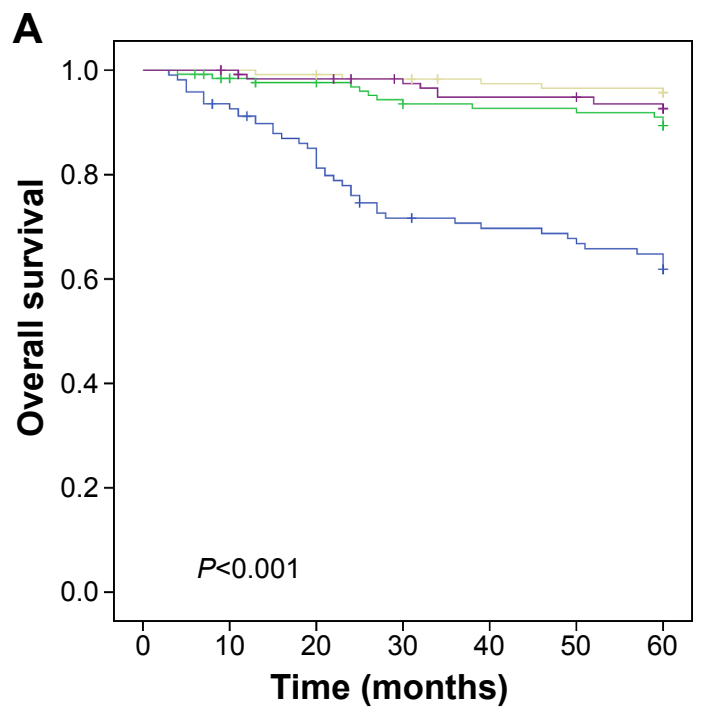

$\mathrm{Q} 4 / \mathrm{Q} 1 ; P=0.001, \mathrm{HR}=2.10)$ and $\mathrm{MFS}(P<0.001, \mathrm{HR}=0.35$ for $\mathrm{Q} 4 / \mathrm{Q} 1 ; P=0.029, \mathrm{HR}=1.61$ for $\mathrm{Q} 4 / \mathrm{Q} 1)$, together with patients' age, $\mathrm{T}$ and $\mathrm{N}$ stage (Table 2 ).

\section{Predictive model for OS and MFS}

To predict the survival of patients with ccRCC after diagnosis, we developed a nomogram by integrating the independent prognostic factors in multivariate analysis (Figure 2). In this nomogram for OS (Figure 2A), higher total points present a poor prognosis. The c-index of the multivariate prognostic model based on age, tumor size, $\mathrm{T}$ and $\mathrm{N}$ stage was 0.729 and improved to 0.817 when the LMR and CRP were supplemented, which showed a better predictive probability of OS than $\mathrm{T}$ and $\mathrm{N}$ stage (c-index 0.677). Calibration plots of this nomogram performed well with the perfect model to predict 3- and 5-year OS (Figure 3A). Meanwhile, the c-index of nomogram for MFS integrating age, $\mathrm{T}$ and $\mathrm{N}$ stage was 0.618 and increased to 0.699 with a combination of LMR and CRP, which showed a better predictive ability of MFS superior to the traditional TNM staging system (Figure 2B). The performance of nomogram was verified by calibration plots (Figure 3B).

\section{Discussion}

To our knowledge, this study was a large sample report to explore two inflammatory factors, which have represented a systemic inflammatory response, and employed the quartile as endpoints to evaluate the prognostic performance of inflammatory response in patients with nonmetastatic ccRCC because of the skewed distribution of LMR and CRP. The elevated CRP and low LMR in the peripheral blood of patients with ccRCC were strongly associated with tumor

B

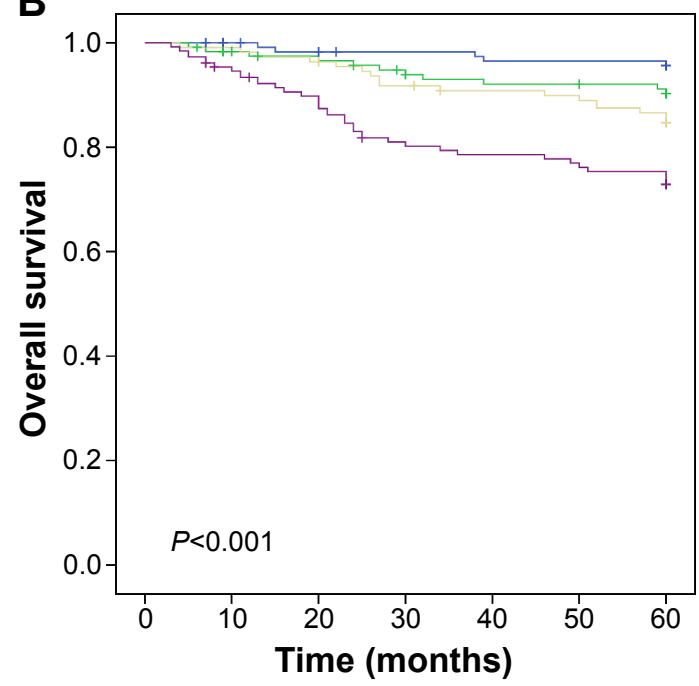

Figure I (Continued) 

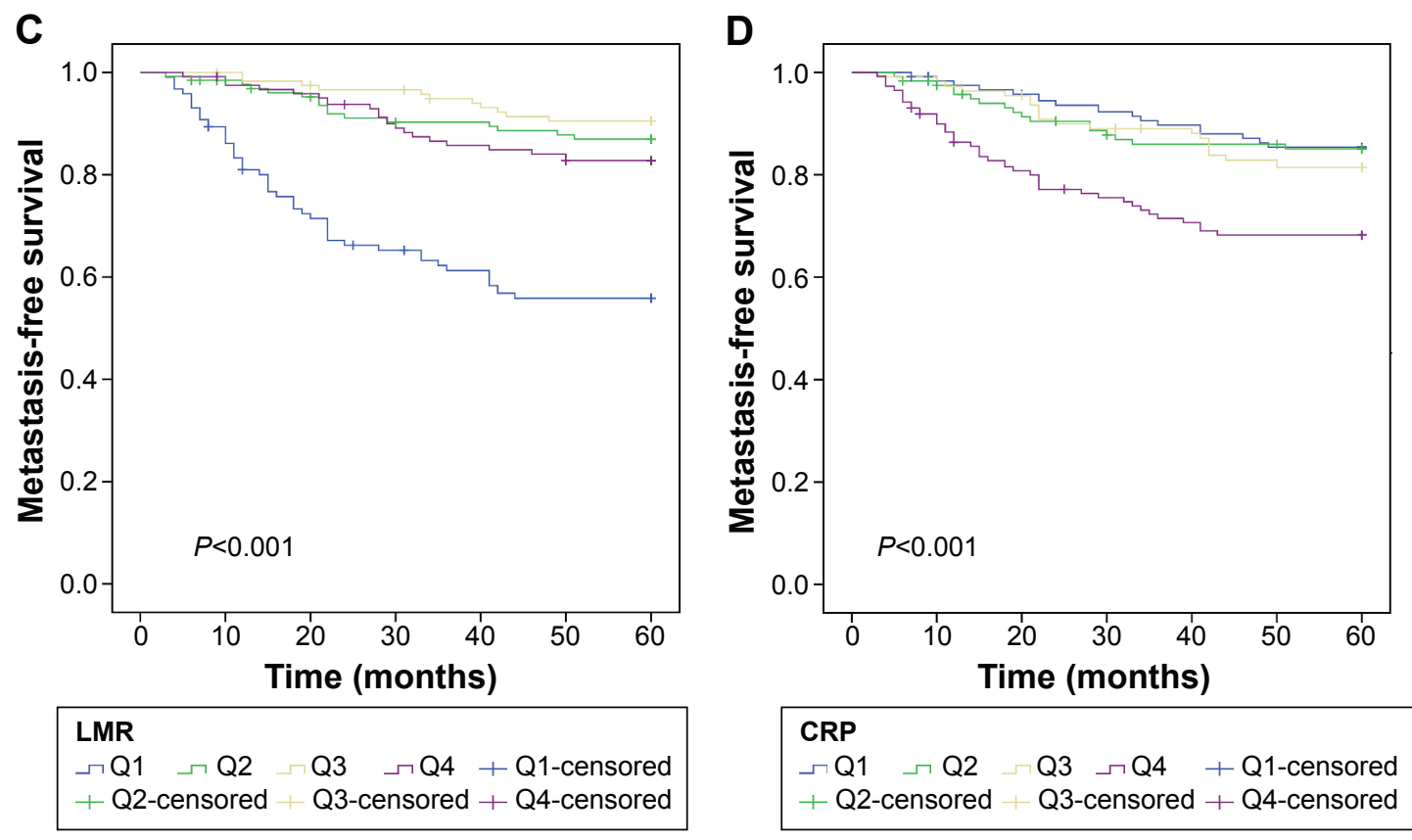

Figure I Kaplan-Meier curves for overall survival (A and B) and metastasis-free survival (C and $\mathbf{D})$ of patients with nonmetastatic clear cell renal cell carcinoma according to LMR (A and $\mathbf{C})$ and CRP (B and $\mathbf{D})$.

Abbreviations: CRP, C-reactive protein; LMR, lymphocyte-to-monocyte ratio; Q, quartile.

Table 2 Univariate and multivariate analyses of clinical characteristics for the prediction of overall and metastasis-free survival

\begin{tabular}{|c|c|c|c|c|c|c|c|c|}
\hline \multirow[t]{3}{*}{ Clinical parameters } & \multicolumn{4}{|c|}{ Overall survival } & \multicolumn{4}{|c|}{ Metastasis-free survival } \\
\hline & \multicolumn{2}{|c|}{$\begin{array}{l}\text { Univariate } \\
\text { analysis }\end{array}$} & \multicolumn{2}{|l|}{ Multivariate analysis } & \multicolumn{2}{|c|}{$\begin{array}{l}\text { Univariate } \\
\text { analysis }\end{array}$} & \multicolumn{2}{|l|}{ Multivariate analysis } \\
\hline & HR & $P$-value & Adjusted HR (95\% CI) & $P$-value & HR & $P$-value & Adjusted HR (95\% Cl) & $P$-value \\
\hline \multicolumn{9}{|l|}{ Sex } \\
\hline Male & 1.00 & & 1.00 & & 1.00 & & 1.00 & \\
\hline Female & 0.70 & 0.053 & $0.98(0.68-\mid .4 I)$ & 0.906 & 0.90 & 0.489 & $1.17(0.86-1.58)$ & 0.315 \\
\hline \multicolumn{9}{|l|}{ Age (years) } \\
\hline$<55$ & 1.00 & & 1.00 & & 1.00 & & 1.00 & \\
\hline$\geq 55$ & 2.28 & $<0.001$ & $1.97(1.37-2.85)$ & $<0.001$ & 1.69 & $<0.001$ & $1.56(1.16-2.09)$ & 0.003 \\
\hline \multicolumn{9}{|l|}{ Smoking* } \\
\hline Yes & 1.00 & & & & 1.00 & & & \\
\hline No & 0.88 & 0.487 & & & 0.94 & 0.701 & & \\
\hline \multicolumn{9}{|l|}{ Drinking* } \\
\hline Yes & 1.00 & & & & 1.00 & & & \\
\hline No & 0.75 & 0.163 & & & 0.78 & 0.146 & & \\
\hline \multicolumn{9}{|l|}{ Tumor size $(\mathrm{cm})$} \\
\hline$<5$ & 1.00 & & 1.00 & & 1.00 & & 1.00 & \\
\hline$\geq 5$ & 4.30 & $<0.001$ & $2.10(1.37-3.20)$ & 0.001 & 2.08 & $<0.001$ & $1.22(0.85-1.76)$ & $0.28 I$ \\
\hline \multicolumn{9}{|l|}{ T stage } \\
\hline $\mathrm{TI}$ & 1.00 & & 1.00 & & 1.00 & & 1.00 & \\
\hline $\mathrm{T} 2$ & 4.23 & $<0.001$ & $2.03(1.27-3.25)$ & 0.003 & 2.38 & $<0.001$ & $1.95(1.36-2.78)$ & $<0.001$ \\
\hline T3-4 & 6.54 & $<0.001$ & $4.72(2.93-7.61)$ & $<0.001$ & 4.14 & $<0.001$ & $4.21(2.83-6.26)$ & $<0.001$ \\
\hline \multicolumn{9}{|l|}{$N$ stage } \\
\hline No & 1.00 & & 1.00 & & 1.00 & & 1.00 & \\
\hline NI & 6.31 & $<0.001$ & $3.68(1.54-8.81)$ & 0.003 & 4.28 & $<0.001$ & $3.13(1.36-7.20)$ & 0.007 \\
\hline \multicolumn{9}{|l|}{ LMR } \\
\hline Quartile I & 1.00 & & 1.00 & & 1.00 & & 1.00 & \\
\hline Quartile 2 & 0.23 & $<0.001$ & $0.26(0.16-0.4 I)$ & $<0.001$ & 0.23 & $<0.001$ & $0.24(0.16-0.36)$ & $<0.001$ \\
\hline Quartile 3 & 0.09 & $<0.001$ & $0.14(0.07-0.27)$ & $<0.001$ & 0.16 & $<0.001$ & $0.17(0.11-0.28)$ & $<0.001$ \\
\hline Quartile 4 & 0.15 & $<0.001$ & $0.23(0.13-0.40)$ & $<0.001$ & 0.30 & $<0.001$ & $0.35(0.24-0.5 I)$ & $<0.001$ \\
\hline \multicolumn{9}{|l|}{ CRP } \\
\hline Quartile I & 1.00 & & 1.00 & & 1.00 & & 1.00 & \\
\hline Quartile 2 & 2.31 & 0.028 & $2.21(1.03-4.74)$ & $0.04 I$ & 1.06 & 0.816 & $1.09(0.67-1.77)$ & 0.715 \\
\hline Quartile 3 & 3.67 & $<0.001$ & 3.11 (I.53-6.35) & 0.002 & 1.29 & 0.275 & $1.23(0.78-1.95)$ & 0.379 \\
\hline Quartile 4 & 7.22 & $<0.001$ & $3.44(1.74-6.80)$ & $<0.001$ & 2.56 & $<0.001$ & $1.61(1.05-2.46)$ & 0.029 \\
\hline
\end{tabular}

Note: $* P>0.05$ in univariate analysis was not further analyzed in multivariate, with the exception of age and sex.

Abbreviations: $\mathrm{Cl}$, confidence interval; CRP, C-reactive protein; HR, hazard ratio; LMR, lymphocyte-to-monocyte ratio. 
A

\section{Points}

Age

Tumor size

$\mathrm{T}$

N

LMR

CRP

Total points

3-year overall survival

5-year overall survival

B

\section{Points}

Age

$\mathrm{T}$

N

LMR

CRP

Total points

3-year metastasis-free survival

5-year metastasis-free survival
$0, \quad 10$

$+30$

2
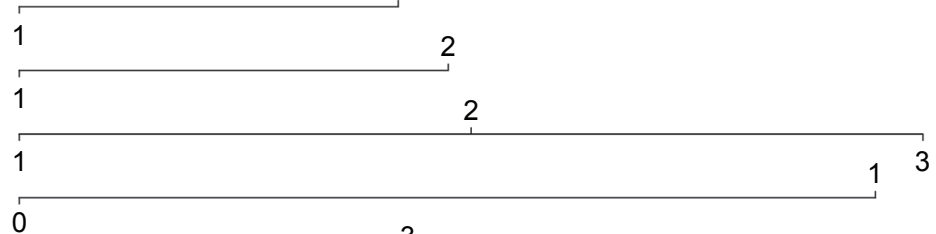

0

42

13

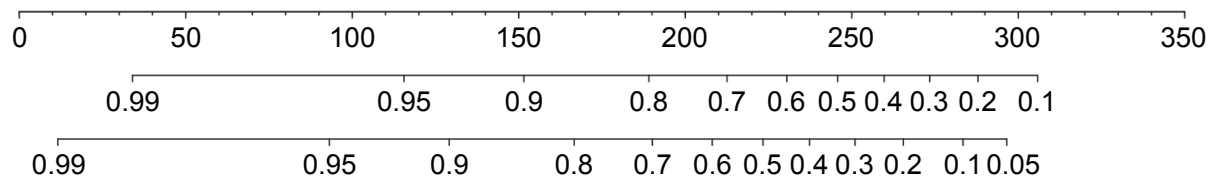

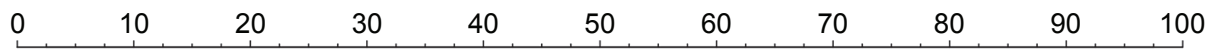

2
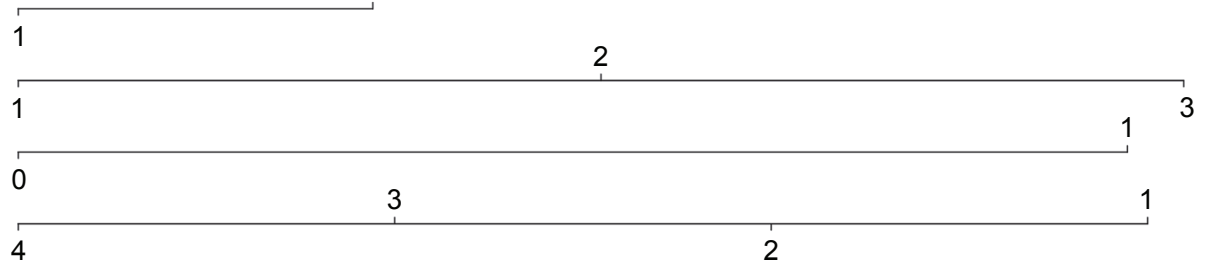

4

2

13

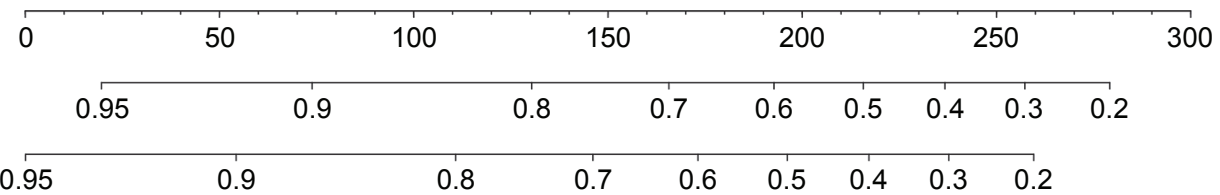

Figure 2 Nomogram for predicting 3- and 5-year overall survival (A) and metastasis-free survival (B) of patients with nonmetastatic clear cell renal cell carcinoma. Abbreviations: CRP, C-reactive protein; LMR, lymphocyte-to-monocyte ratio.

progression and worse outcome after treatment. Although inflammatory biomarkers have been widely investigated in a variety of cancers, ${ }^{15,19}$ the impact of inflammatory markers on the outcome of patients with ccRCC remains confusing. Hence, the purpose of this study was to evaluate the prognosis of 958 patients with ccRCC based on inflammatory biomarkers (LMR and CRP) in the peripheral blood, and we developed a nomogram to improve the predictive accuracy and evaluated its performance by an internal validation. Peripheral blood count tests are routinely conducted without additional effort in all patients with cancer; they are simple, cheap, and reproducible factors of the inflammatory response as well as prognostic indicators. Therefore, our study may provide critical prognostic information that augments the analyses of traditional clinical characteristics.
Links between inflammation and cancer were first reported in the nineteenth century, and contemporary studies show a general viewpoint that the inflammatory response has a necessary role in carcinogenesis. ${ }^{20}$ Cancer-associated inflammation is characterized with the infiltration of inflammatory cells and secretion of inflammatory mediators into tumor tissues, cancer remodeling, repair, and angiogenesis. ${ }^{21}$ Furthermore, inflammation is susceptible to induce protumorigenic microenvironment changes, which can aggravate tumor progression. ${ }^{22}$ Accordingly, the complex interactions of inflammatory cells and mediators in tumor microenvironment may be reflected in the peripheral circulation.

Recent data have reported the prognostic value of LMR in patients with nonmetastatic ccRCC ${ }^{16,18}$ and ccRCC. ${ }^{17}$ 

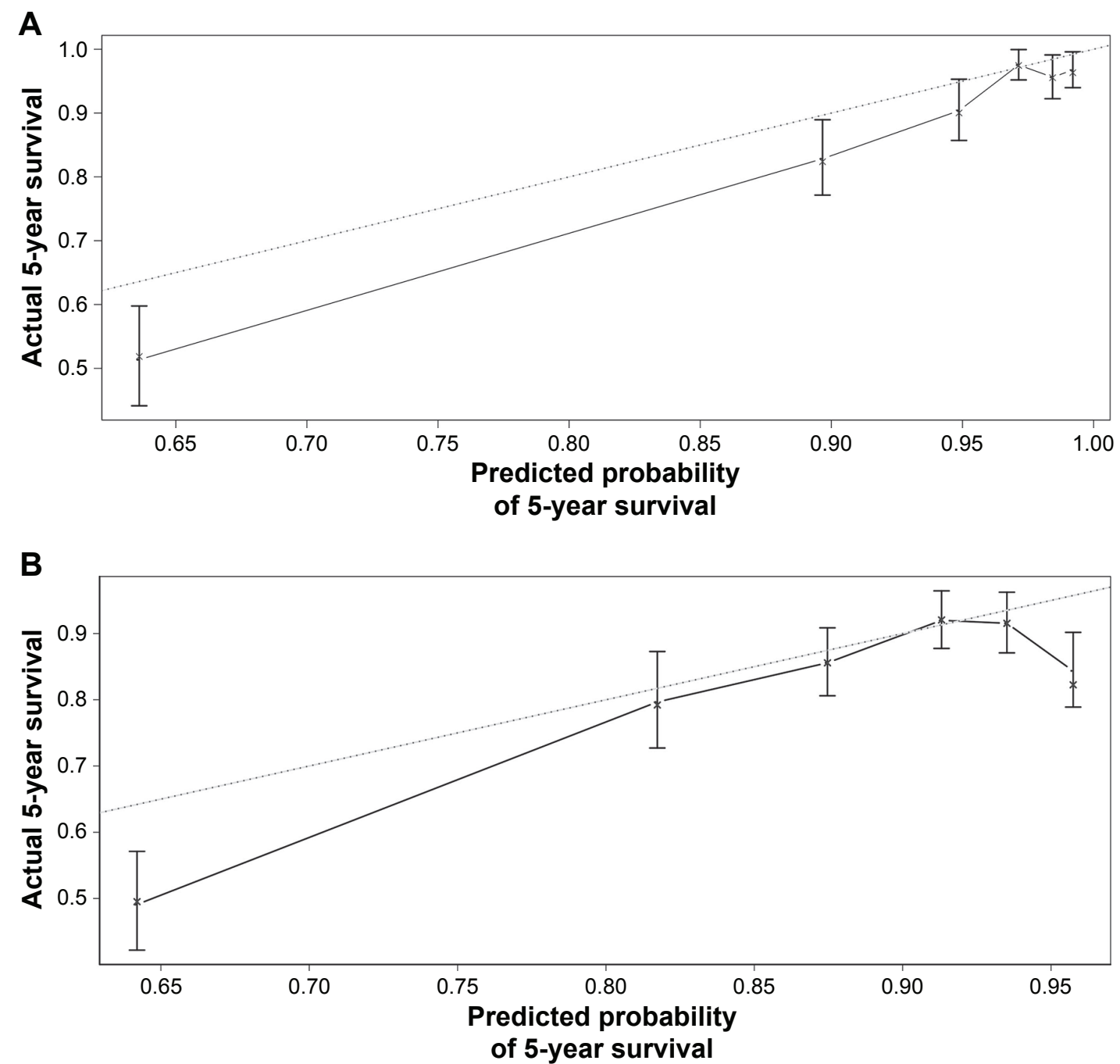

Figure 3 Calibration plot of the nomogram for 5-year OS (A) and MFS (B).

Notes: The solid line shows the performance of the observed nomogram. The gray dashed line indicates the performance of an ideal nomogram. $X$ axis is the bootstrapped corrected evaluation of nomogram with I,000 resamples. Vertical bars represent $95 \%$ confidence interval. It seems that the nomograms accurately predict the 5 -year OS (A) and MFS (B). (A) $n=958 ; d=133 ; P=6$; I 50 subjects per group; $X$ - resampling optimism added, $B=1,000$; comparison between nomogram-predicted probability of OS ( $x$-axis) and the actual 5 -year survival (y-axis). (B) $n=958 ; d=188 ; P=5$; 150 subjects per group; $X$ - resampling optimism added, $B=I, 000 ;$ comparison between nomogram-predicted probability of MFS ( $x$-axis) and the actual 5 -year survival ( $y$-axis).

Abbreviations: MFS, metastasis-free survival; OS, overall survival.

Moreover, in a meta-analysis by Zhou et al, ${ }^{23}$ high CRP was obviously associated with worse outcomes in patients with RCC. However, the prognostic performance of integrating these two factors and laboratory parameters into the traditional TNM staging system remains obscure in nonmetastatic ccRCC. In this study, we demonstrated that CRP and LMR were independent prognostic factors of OS and MFS for patients with nonmetastatic ccRCC. Moreover, integrated variables based on CRP and LMR significantly improved the predictive accuracy for OS and MFS. Hutterer et al ${ }^{18}$ first identified that a low LMR reduced cancer-specific survival, OS, and MFS in 678 patients with nonmetastatic ccRCC, and is an independent predictor of worse cancer-specific survival but not of MFS or OS after adjustment. Inconsistent with the earlier findings, our study showed the prognostic role of low LMR as an independent predictor of short OS and MFS for patients with nonmetastatic ccRCC in Asia. Different cutoff values and statistical methods may give rise to contradictory results for OS and MFS. In addition, Chang et $\mathrm{al}^{16,17}$ performed two studies to explore the prognostic value of inflammatory response markers in patients with ccRCC or nonmetastatic ccRCC from a single center in the People's Republic of China. They presented an interesting conclusion that low LMR is an independent prognostic indicator of recurrence-free survival and OS in 430 patients with nonmetastatic $\mathrm{ccRCC}^{16}$ as well as that of OS in 441 ccRCC patients who underwent nephrectomy. ${ }^{17}$ Moreover, they created a novel prognostic score based on serum albumin 
and LMR, which was integrated into traditional clinical parameters to improve survival prediction of patients with ccRCC. ${ }^{17}$ Elevated CRP has been reported to be associated with poor outcome in several kinds of cancers. ${ }^{21}$

A systemic inflammatory response may cause high levels of CRP in serum, and many inflammatory mediators are derived from tumor tissue. Carcinogenesis and tumor progression can be activated by the inflammatory microenvironment of the tumor. ${ }^{24}$ We demonstrated that high CRP was an independent predictor of diminished OS and MFS. Moreover, both CRP and LMR were incorporated into the traditional TNM staging system and other clinical variables. Accordingly, this is the first multicenter study integrating LMR and CRP into the traditional clinical characteristics to supplement outcome prediction of 958 patients with ccRCC.

The strength of this report is the large, multicenter sample size and integration of LMR, CRP, and traditional clinical features. However, several limitations should be acknowledged. First, selection bias may be derived from the retrospective design of our study. A prospective study is urgently needed to identify the results of our study. Second, the heterogeneities of treatment are not omitted because of the multicenter study. Third, due to a lack of patients with metastasis, we could not assess the correlation between two inflammatory markers and metastatic disease.

\section{Conclusion}

In summary, our study suggests that LMR and CRP are independent predictors of OS and MFS in patients with nonmetastatic ccRCC after treatment and should be incorporated into traditional clinical features to improve the predictive probability of survival of patients with nonmetastatic ccRCC.

\section{Ethical standards}

The study was approved by the Medical Ethics Committee of Jiangyin People's Hospital, Hangzhou First People's Hospital, and Zhejiang Cancer Hospital, respectively.

\section{Author contributions}

Protocol development, HH, WKX; data collection, WKX, XW, THY, YW, XJY; data analysis, WKX, XW; manuscript writing/editing, WKX, HH; supervision, HH. All authors contributed toward data analysis, drafting and revising the paper, and agree to be accountable for all aspects of the work.

\section{Disclosure}

The authors report no conflicts of interest in this work.

\section{References}

1. Parkin DM, Bray F, Ferlay J, Pisani P. Global cancer statistics, 2002. CA Cancer J Clin. 2005;55(2):74-108.

2. Cheville JC, Lohse CM, Zincke H, Weaver AL, Blute ML. Comparisons of outcome and prognostic features among histologic subtypes of renal cell carcinoma. Am J Surg Pathol. 2003;27(5):612-624.

3. Rini BI, Campbell SC, Escudier B. Renal cell carcinoma. Lancet. 2009;373(9669):1119-1132.

4. Siegel R, Naishadham D, Jemal A. Cancer statistics, 2013. CA Cancer J Clin. 2013;63(1):11-30.

5. Ljungberg B, Campbell SC, Choi HY, et al. The epidemiology of renal cell carcinoma. Eur Urol. 2011;60(4):615-621.

6. Edge SB, Compton CC. The American Joint Committee on Cancer: the 7 th edition of the AJCC cancer staging manual and the future of TNM. Ann Surg Oncol. 2010;17(6):1471-1474.

7. Shariat SF, Xylinas E. Biomarkers in personalised treatment of renalcell carcinoma. Lancet Oncol. 2012;13(8):751-752.

8. Mantovani A. Cancer: inflaming metastasis. Nature. 2009;457(7225): $36-37$.

9. Roxburgh CS, McMillan DC. Role of systemic inflammatory response in predicting survival in patients with primary operable cancer. Future Oncol. 2010;6(1):149-163.

10. Pichler M, Hutterer GC, Stoeckigt C, et al. Validation of the pretreatment neutrophil-lymphocyte ratio as a prognostic factor in a large European cohort of renal cell carcinoma patients. Br J Cancer. 2013;108(4):901-907.

11. Park YH, Ku JH, Kwak C, Kim HH. Post-treatment neutrophil-tolymphocyte ratio in predicting prognosis in patients with metastatic clear cell renal cell carcinoma receiving sunitinib as first line therapy. Springerplus. 2014;3:243.

12. Hu K, Lou L, Ye J, Zhang S. Prognostic role of the neutrophillymphocyte ratio in renal cell carcinoma: a meta-analysis. BMJ Open. 2015;5(4): e006404.

13. Deng Q, He B, Liu X, et al. Prognostic value of pre-operative inflammatory response biomarkers in gastric cancer patients and the construction of a predictive model. $J$ Transl Med. 2015;13:66.

14. Ying HQ, Deng QW, He BS, et al. The prognostic value of preoperative NLR, d-NLR, PLR and LMR for predicting clinical outcome in surgical colorectal cancer patients. Med Oncol. 2014;31(12):305.

15. Song YJ, Wang LX, Hong YQ, et al. Lymphocyte to monocyte ratio is associated with response to first-line platinum-based chemotherapy and prognosis of early-stage non-small cell lung cancer patients. Tumour Biol. Epub 2015 Nov 11.

16. Chang Y, Fu Q, Xu L, et al. Prognostic value of preoperative lymphocyte to monocyte ratio in patients with nonmetastatic clear cell renal cell carcinoma. Tumour Biol. Epub 2015 Oct 27.

17. Chang Y, An H, Xu L, et al. Systemic inflammation score predicts postoperative prognosis of patients with clear-cell renal cell carcinoma. Br J Cancer. 2015;113(4):626-633.

18. Hutterer GC, Stoeckigt C, Stojakovic T, et al. Low preoperative lymphocyte-monocyte ratio (LMR) represents a potentially poor prognostic factor in nonmetastatic clear cell renal cell carcinoma. Urol Oncol. 2014;32(7):1041-1048.

19. Jia W, Wu J, Jia H, et al. The peripheral blood neutrophil-to-lymphocyte ratio Is superior to the lymphocyte-to-monocyte ratio for predicting the long-term survival of triple-negative breast cancer patients. PLoS One. 2015;10(11):e0143061.

20. Balkwill F, Mantovani A. Inflammation and cancer: back to Virchow? Lancet. 2001;357(9255):539-545.

21. Mantovani A, Allavena P, Sica A, Balkwill F. Cancer-related inflammation. Nature. 2008;454(7203):436-444. 
22. Chechlinska M, Kowalewska M, Nowak R. Systemic inflammation as a confounding factor in cancer biomarker discovery and validation. Nat Rev Cancer. 2010;10(1):2-3.

23. Zhou L, Cai X, Liu Q, Jian ZY, Li H, Wang KJ. Prognostic role of C-reactive protein in urological cancers: A meta-analysis. Sci Rep. 2015;5:12733.
24. Gueron G, De Siervi A, Vazquez E. Advanced prostate cancer: reinforcing the strings between inflammation and the metastatic behavior. Prostate Cancer Prostatic Dis. 2012;15(3):213-221.

\section{Publish your work in this journal}

OncoTargets and Therapy is an international, peer-reviewed, open access journal focusing on the pathological basis of all cancers, potential targets for therapy and treatment protocols employed to improve the management of cancer patients. The journal also focuses on the impact of management programs and new therapeutic agents and protocols on

\section{Dovepress}

patient perspectives such as quality of life, adherence and satisfaction. The manuscript management system is completely online and includes a very quick and fair peer-review system, which is all easy to use. Visit http://www.dovepress.com/testimonials.php to read real quotes from published authors.

\footnotetext{
Submit your manuscript here: http://www.dovepress.com/oncotargets-and-therapy-journal
} 